\title{
Combination of high-intensity focused ultrasound irradiation and hydroxyapatite nanoparticle injection to injure normal goat liver tissue in vivo without costal bone incision
}

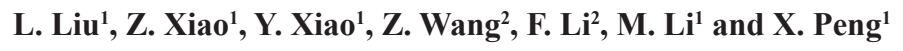 \\ 'Department of Ultrasound, \\ The First Affiliated Hospital of Chongqing Medical University, \\ China \\ ${ }^{2}$ Department of Biomedical Engineering, Chongqing Medical University, \\ China \\ Corresponding author: L. Liu \\ E-mail: liuliping_1lp@126.com
}

Genet. Mol. Res. 13 (4): 8301-8308 (2014)

Received June 21, 2013

Accepted February 10, 2014

Published October 20, 2014

DOI http://dx.doi.org/10.4238/2014.October.20.6

\begin{abstract}
The aims of this study were to evaluate the in vivo safety of intravenous nano-hydroxyapatite (nano-HA), to explore how nanoHA might influence the effects of high-intensity focused ultrasound (HIFU) on normal liver tissue, and to investigate whether intravenous nano-HA could enhance HIFU for hepatocellular carcinoma ablation in a goat model. The present study, for the first time, indicated that the delivery of abundant nano-HA into the body over short periods of time could be assembled by the hepatic reticuloendothelial system, subsequently leading to a rapid rise of ultrasound-induced overheating, and ultimately resulting in enlargement of the coagulation necrotic area for ablated hepatocellular carcinoma in goats both in vivo and ex vivo. On the other hand, therapeutic doses of nano-HA were much lower than the lethal dose, and consequently presented transient and mild
\end{abstract}


abnormalities of hepatic enzymes and renal function during the first 24 $\mathrm{h}$ after nano-HA injection. These results suggested that the combined application of nano-HA and HIFU is potentially a more effective alternative option compared to surgery for hepatocellular carcinoma local ablation in a safe and feasible manner.

Key words: Nano-hydroxyapatite; Hepatocellular carcinoma; High-intensity focused ultrasound; Rabbit model

\section{INTRODUCTION}

High-intensity focused ultrasound (HIFU) therapy is a new non-invasive type of cancer treatment (Grenier et al., 2009). However, its application is limited when faced with profound-depth or large-sized tumors. To resolve this problem, altering the sound environment of the targeted regions seems to be the only safe, effective, and non-invasive method. Thereby, extrinsic contrasts have received more and more attention to change acoustic characteristics of the targeted region and ultimately enhance HIFU's ablative effectiveness while minimizing damage to normal adjacent tissues. Inorganic hydroxyapatite nanoparticles composed of calcium phosphate (nano-HA) are attracting considerable interest for disease-specific imaging contrast due to its ease of synthesis, optimal control of physicochemical properties, and biocompatibility, as well as its smaller size than red blood cells to ensure its free transfusion in the blood cycle in vivo (Lock et al., 2012; Hu et al., 2012)

Furthermore, a notable obstacle of the costal bone's barriers can attenuate HIFU's ablative effectiveness for hepatic solid tumors. Thereby, invasive surgeries for costal bone incision have to be commonly performed prior to non-invasive HIFU applications. To mimic the human anatomical structure of the hepatic region and to ultimately solve the paradoxical issue above, the aims of this study were to evaluate the in vivo safety and feasibility of the combination of HIFU radiated ablation and nano-HA injection to damage normal goat liver tissue in vivo without costal bone incision.

\section{MATERIAL AND METHODS}

\section{Animals and materials}

The animal study was approved by the Chongqing Experimental Animal Committee (Chongqing, China). All the 30 Nanjiang goats of mixed gender, excluding pregnant goats, were provided by the Experimental Animals Center of Chongqing Medical University, and were kept in a controlled environment with free access to food and water. The average age was 7.2 months ranging from 6 to 10 months, and the average weight was $21.5 \pm 5.36 \mathrm{~kg}$ ranging from 16.5 to $27.2 \mathrm{~kg}$. Goats were randomly divided into two equal groups as the nano-HA and the control group ( $\mathrm{N}=15$ each), and were then subdivided into two equal groups as the dottargeted irradiated subgroup $(\mathrm{N}=10)$ and the line scanning subgroup $(\mathrm{N}=5)$.

The needle-like nano-HA composites, approximately $12-19.33 \mathrm{~nm}$ in diameter and 42.6-183.2 $\mathrm{nm}$ in length, were provided by the Sichuan University Biomaterials Engineering Research Center (Lot No. 3030002). The experimental nano-HAs were diluted with $0.9 \%$ 
saline to a $20 \mathrm{~g} / \mathrm{L}$ concentration. Lecithin, sodium carboxymethyl cellulose, glycerine, acetic acid glacial saline, calcium nitrate, ammonium phosphate, and arginine were purchased from the Sigma Corporation (St. Louis, MO, USA) and the Shanghai Chemistry Reagent Company (Shanghai, China).

\section{Instruments}

The JC-A ultrasonic therapeutic apparatus, the TES1310 contact thermometer (XMZ101G; Taiwan), and the Hitachi H-600 transmission electron microscope were provided by the Institute of Ultrasonic Engineering in Medicine of Chongqing Medical University. NanoHA suspensions in various solvents were sonicated with an XL2020 sonicator microtip (Heat Systems; Farmindale, NY, USA). Biochemical indicators of multiple organ function were analyzed using a Beckman CX7 analyzer with Beckman reagents (High Wycombe; UK).

\section{Influence of intravenous nano-HA in liver damage by HIFU in a goat model without costal bone incision}

All goats were fasted for $24 \mathrm{~h}$ before HIFU treatment. The nano-HA group was quickly injected with $20 \mathrm{mg} / \mathrm{kg}$ nano-HAs and $2 \mathrm{~mL} 0.9 \%$ saline was then injected in the auricular vein, whereas the control group was injected with $1 \mathrm{mg} / \mathrm{kg}$ normal saline. In the morning of HIFU treatment, all goats received a quick muscular infusion of $0.01 \mathrm{~mL} / \mathrm{kg}$ pig anesthetic. Skin preparation for the right chest and abdomen regions was sequentially performed by shedding with razors and $8 \%$ sodium sulfide, degreasing with hydrous ethanol (about $95 \%$ ethanol and 5\% water), and abdominal degassing with the off-gas pump. The goats were then fixed to HIFU instruments in the right lateral position to ensure that the hepatic regions were completely soaked in the cycling degassing water, and the HIFU's focus was located at the goat's liver guided by ultrasound.

The parameters of HIFU for liver damage were as follows: up to $200 \mathrm{~W}$ generator power, $0.7 \mathrm{mHz}$ frequency, $135 \mathrm{~mm}$ focal length, and $40 \mathrm{~mm}$ radiated length. The scanning rate was $2 \mathrm{~mm} / \mathrm{s}$ with $2 \mathrm{~s}$ durations per time and 8-10-s intervals. The 4-5 irradiated planes were parallel to the tangent plane of the costal bones and the intercostal space to avoid the costal bones' obstacle as much as possible. Two to three dots or lines of each irradiated plane, avoiding large vessels and the gall bladder as much as possible, were chosen as the targets of HIFU treatment.

Forty-eight to seventy-two hours after HIFU treatment, all experimental goats received median laparotomies to expose the liver after a quick muscular infusion of $0.01 \mathrm{~mL} / \mathrm{kg}$ pig anesthetic. Liver tissues were fixed with 5\% glutaric dialdehyde for scanning electronic microscopy and with $10 \%$ paraformaldehyde for light microscopy. All goats were then sacrificed by a quick infusion of $30 \mathrm{~mL}$ air in the auricular veins. Subsequently, the whole livers were taken out. Volumes $\left(\mathrm{V} ; \mathrm{mm}^{3}\right)$ of coagulation necrotic loci under HIFU were calculated as in (Equation 1):

$$
\mathrm{V}=\frac{\pi}{6} a b h
$$

(Equation 1)

where $a$ indicates the major axis, $b$ is the minor axis, and $h$ is the depth perpendicular to the 
plane of the sound channel. The energy efficiency factor (EEF) was applied and calculated as in (Equation 2):

\section{巴一=nPT/V}

(Equation 2)

where $\eta$ indicates the energy efficiency coefficient, $P$ is the generator power, $T$ is the pulse duration time, and $V$ is the volume of the coagulation necrotic loci from (Equation 1).

\section{Histological examination}

After overnight fixation, part of the damaged hepatic tissues were trimmed and cut through at the widest margin. The tissues were embedded in paraffin and sectioned in 5- $\mu \mathrm{m}$ increments. The sections were made perpendicular to the anterior-posterior axes. Three sections were placed on a slide and stained with hematoxylin and eosin (HE), as well as periodic acid-Schiff (PAS). Of the three sections on any one slide, the sections with the widest tissue parenchyma were used for assessment. The fine structures of the liver were observed by electron microscopy.

\section{Statistical analysis}

Data were analyzed using the Statistical Package for the Social Sciences, version 13.0 (SPSS Inc.; Chicago, IL, USA). We used the two-tailed unpaired Student $t$-test for comparisons between groups and subgroups. A value of $\mathrm{P}<0.05$ was considered to be statistically significant.

\section{RESULTS}

\section{Efficacy of combination HIFU dot irradiation and nano-HA injection to injure normal goat liver tissue in vivo without costal bone incision}

Upon administration of HIFU treatment, coagulated necrotic lesions were clearly observed using both ultrasound imaging and by performing animal vivisection. Primary lesions of small-size punctiforms were observed in the HIFU control group, whereas multiform laminated lesions were found in the nano-HA and HIFU combination group as shown in Figure 1.

As shown in Table 1, within $150 \mathrm{~s}$ dot-irradiated time, there were 12 dots of the total targeted 30 dots $(40.00 \%)$ in the nano-HA and HIFU combination group, while only 5 coagulative necrotic dots of the total targeted 32 dots $(15.63 \%)$ were observed in the control group $(\mathrm{P}<0.05)$. Furthermore, a significantly lower EEF was found in the nano-HA and HIFU combination group $(\mathrm{P}=0.000)$, corresponding to almost one fourth of that in the control group. This result indicated that nano-HA might improve HIFU treatment by nearly 3.5 -fold to injure normal goat liver tissue in vivo without costal bone incision. 


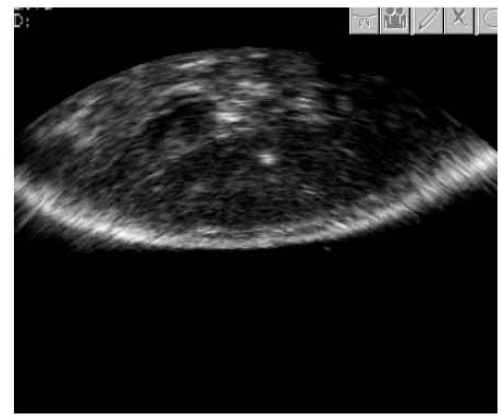

A

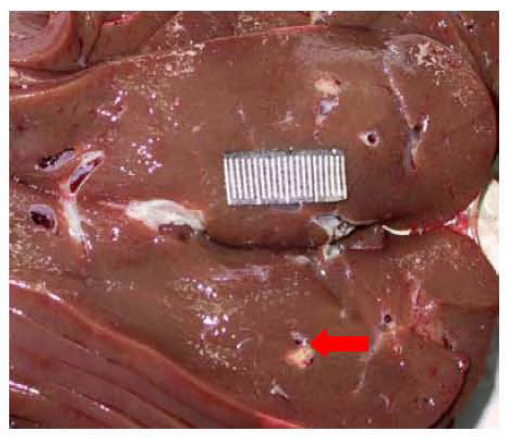

C

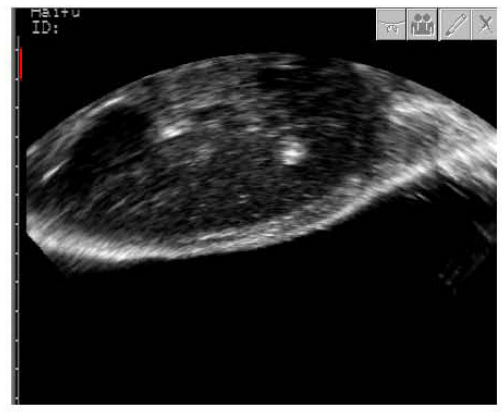

B

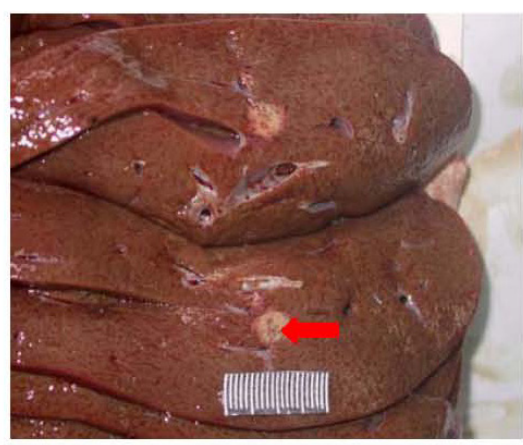

Figure 1. A. Ultrasound image of one goat of control group $160 \mathrm{~s}$ after high-intensity focused ultrasound (HIFU) dot exposure. B. Ultrasound image of one goat of nano-hydroxyapatite (nano-HA) group $120 \mathrm{~s}$ after HIFU dot exposure. C. Coagulative necrosis in round liver of of control group $160 \mathrm{~s}$ after HIFU dot exposure. D. Coagulative necrosis in rounddot shape ball shape in the goat liver of nano-HA group $120 \mathrm{~s}$ after HIFU dot exposure.

Table 1. Effects of nano-HA to the effection of HIFU with dot exposure to the goat liver.

\begin{tabular}{lcccc}
\hline & $\mathrm{N}$ & Mean exposure time $(\mathrm{s})$ & Mean volume of necrosis $\left(\mathrm{mm}^{3}\right)$ & $\mathrm{Mean}$ EEF $\left(\mathrm{J} / \mathrm{mm}^{3}\right)$ \\
\hline Control group & $5(32)$ & $154.00 \pm 7.84$ & $127.38 \pm 61.76$ & $221.10 \pm 111.17$ \\
HA group & $12(30)$ & $140.17 \pm 25.38$ & $475.19 \pm 124.34$ & $61.78 \pm 10.04$ \\
\hline
\end{tabular}

nano-HA = nano-hydroxyapatite; HIFU = high-intensity focused ultrasound; EEF = energy efficiency factor.

\section{Efficacy of combination HIFU line irradiation and nano-HA injection to injure normal goat liver tissue in vivo without costal bone incision}

No obvious coagulation necrotic lesions were observed in either the control or the nanoHA group during the 150-200 s of HIFU line irradiation. However, in addition to $150 \mathrm{~s}$ HIFU line irradiation, a large amount of burnables and thermal curing injuries were found in the sound energy channels passing through the goats' skin, costal bones, and hepatic capsules (Figure 2). 


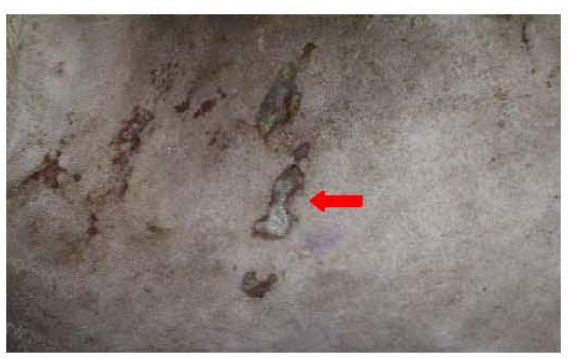

A

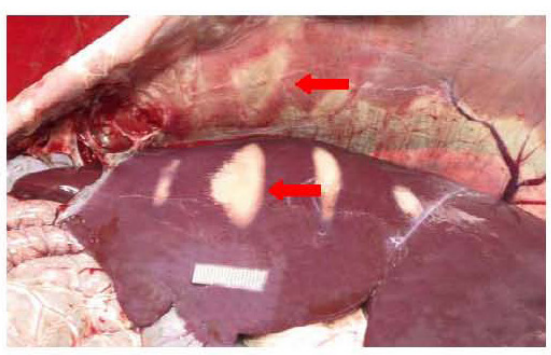

B

Figure 2. A. Burns on the goat skin of the control group $150 \mathrm{~s}$ after high-intensity focused ultrasound (HIFU) dot exposure. B. Burns on the ribs and hepato-membrane of the control group $150 \mathrm{~s}$ after HIFU dot exposure.

\section{Histological examination}

Pathological changes were characterized by coagulation necrosis in both the control and nano-HA groups. In other words, the hepatic tissue exhibited destruction of the hepatic plate and cord structure, and the hepatic cells showed nuclear shrinkage, nuclear fragmentation, karyolysis, vesicular cytoplasm, apoptotic bodies, a vague cell shape, and so on under light microscopic observation. The main characteristics observed under electron microscopy were as follows: the hepatic cells exhibited cellular structure collapse, organelle fragmentation, distended rough endoplasmic reticula, a remarkably decreased number of rough endoplasmic reticula and degranulized, dilated, and vacuolized smooth endoplasmic reticula, swollen and deformed mitochondria, destroyed mitochondrial structure, a remarkable decrease in glycogenosomes, and even disappearance of glycogenosomes.

\section{DISCUSSION}

To date, more than 4000 patients with solid tumors have received HIFU treatment and obtained satisfactory therapeutic effects. However, HIFU application is limited when facing neoplasms of large size or profound depth, which is related to the obvious attenuation of acoustic energy during the transmission course (Tachikawa et al., 1999). To resolve this issue, three aspects could be taken into consideration: 1) increase the HIFU therapeutic dose, such as acoustic power; 2) cut off the costal bones on the acoustic channel; 3) change the acoustic environment of the targeted region. Of note, there is a limit to the extent to which HIFU's therapeutic dose can be increased due to irradiated damage to the normal tissue, and cutting the costal bones eliminates the main non-invasive advantage of HIFU treatment. Thereby, the only reasonable available option is to alter the tissues' acoustic environment in order to advance the deposition of ultrasound energy.

As a new drug and gene delivery carrier, medical polymer nanoparticles appear to be very promising and have been widely studied. Nanoparticles possess several advantages as a delivery system over microparticles, including their subcellular size, targeted surfaces, uptake by cells through the tissue matrix, easy penetration into the arterial wall without causing trauma, and their ability to cross the blood-brain barrier. On the other hand, hydroxylapatite (HA), a crystalline calcium phosphate with the small length of 1-100 nm and large specific 
surface area, is a primary inorganic component of human bones and teeth with satisfactory bioactivity (Lee et al., 2012). Because artificial nano-HA particles are very similar to demic HA, nano-HAs possess advantageous biocompatibility and bioactivity. Currently, nano-HAs have been widely applied in the medical field including as alternative bone, as nanoparticle drug carriers, and so on. Furthermore, nano-HA particles can exert anti-tumor effects by themselves. For example, nano-HA particles were reported to significantly inhibit the growth of the human hepatoma cell line BEL-7402 ex vivo by inducing tumorous apoptosis and decreasing proliferation while exerting no or only mild damage to human normal hepatic cells. Herein, we proposed the hypothesis that nano-HAs might be an optimal vector to alter the tissues' acoustic environment in order to enhance the tumor ablative effects of HIFU.

On the other hand, previous reports showed that effective coagulation necrotic damage could occur as a result of HIFU treatment based on ex vivo studies in bovine livers or small animals (Owen et al., 2010). However, the acoustic environmental characteristics of ex vivo bovine livers is significantly different from that of normal in vivo bovine livers due to the loss of blood circulation and ischemia-related hepatic damage. Moreover, there was a relatively small attenuation of acoustic energy when HIFU was passed through the hepatic regions of rabbits and other small animals because of the thin skin of their abdomens and the lack of obvious costal bone obstacles. Accordingly, HIFU therapy did not appear to be suitable for big animals and humans. Therefore, the present study adopted the goat model, which possesses thick skin, subcutaneous fat, and costal bone obstacles, to mimic the human acoustic environment for hepatic treatment with a combination of HIFU and nano-HA.

The present study demonstrated that nano-HA particles could be an effective and safe vector for changing the acoustic environment of hepatic tissues (Meng et al., 2008). Intriguingly, in the present study, coagulative necrosis was observed by dot irradiation both with and without the use of nano-HAs, whereas compared to the use of HIFU irradiation on its own, a nearly 3.5 -fold enhanced effect could be obtained by the addition of nano-HAs in the absence of a real-time acoustic image monitor. Nevertheless, no coagulative necrosis was observed from the 150-200 s line irradiation with or without the addition of nano-HAs in the presence of severe damage of the skin, costal bones, and hepatic capsular through the acoustic channel, which significantly decreased the HIFU energy before its arrival at the irradiated focus.

Different enhancements of HIFU's irradiation by nano-HAs may be related to different HIFU therapeutic models. During dot irradiation, light beams passed through the intercostal space with a fixed acoustic channel and focus. Consequently, irradiated energy at the focus region could be accumulated to the threshold value, resulting in coagulative necrosis, although some acoustic energy decayed through the skin and bones along the transmission path. However, although the irradiated range of the acoustic channel is wider by line irradiation than by dot irradiation, the irradiated pointers are always moving in line irradiation, and consequently, only transient therapeutic time can be obtained at the line-targeted regions. Therefore, it is challenging to accumulate the HIFU dose to the therapeutic threshold value with the lineirradiated model. The enhanced effects of nano-HAs have been confirmed, but are still limited during line irradiation. This might indicate that other nano-particles with bigger non-linear parameters than nano-HAs might be more helpful to assist HIFU line irradiation. The data obtained in the present study is only preliminary, but nonetheless revealed that the combination of HIFU irradiation and hydroxyapatite nano-particle injection is a feasible option to injure normal goat liver tissue in vivo without costal bone incision, and the optimal parameters will be determined in future studies. 


\section{ACKNOWLEDGMENTS}

Research supported by the National Key Clinical Specialties Construction Program of China (\#2013544) and the Project of the Medical Science and Technology Foundation of Chongqing Health Commission (\#2011-2-100).

\section{REFERENCES}

Grenier N, Quesson B, de Senneville BD, Trillaud H, et al. (2009). Molecular MR imaging and MR-guided ultrasound therapies in cancer. JBR-BTR 92: 8-12.

Hu NM, Chen Z, Liu X, Liu H, et al. (2012). Mechanical properties and in vitro bioactivity of injectable and self-setting calcium sulfate/nano-HA/collagen bone graft substitute. J. Mech. Behav. Biomed. Mater. 12: 119-128.

Lee WH, Zavgorodniy AV, Loo CY and Rohanizadeh R (2012). Synthesis and characterization of hydroxyapatite with different crystallinity: effects on protein adsorption and release. J. Biomed. Mater. Res. A 100: 1539-1549.

Lock J, Nguyen TY and Liu H (2012). Nanophase hydroxyapatite and poly (lactide-co-glycolide) composites promote human mesenchymal stem cell adhesion and osteogenic differentiation in vitro. J. Mater. Sci. Mater. Med. 23: 2543-2552.

Meng YH, Tang CY, Tsui CP and Chen da Z (2008). Fabrication and characterization of needle-like nano-HA and HA/ MWNT composites. J. Mater. Sci. Mater. Med. 19: 75-81.

Owen NR, Bouchoux G, Seket B, Murillo-Rincon A, et al. (2010). In vivo evaluation of a mechanically oscillating dualmode applicator for ultrasound imaging and thermal ablation. IEEE Trans. Biomed. Eng. 57: 80-92.

Tachikawa D, Inada S, Kotoh T, Futami K, et al. (1999). An evaluation of malignancy and prognostic factors based on mode of lymph node metastasis in esophageal carcinoma. Surg. Today 29: 1131-1135. 